\title{
Organic Mental Disorder
}

National Cancer Institute

\section{Source}

National Cancer Institute. Organic Mental Disorder. NCI Thesaurus. Code C34870.

Any decrease in mental functioning in which the cause is not attributable to psychiatric illness, which includes damage from physical trauma, anoxic injury, damage from chemicals or toxins, infection, cancer, and degenerative diseases, including Alzheimer's disease, Huntington's disease, Parkinson's disease, and Creutzfeldt-Jakob disease. 